\title{
Artículos
}

\section{Así anda el mundo de mal}

\author{
Aquiles Montoya
}

Resumen
Para comprender la problemática actual del mundo es preciso co-
nocer cuáles son las principales tendencias que están delineando el
futuro previsible. Hoy más que nunca estamos viviendo el inicio
de una fase de transición hacia una sociedad mundial desgarrada por
la pobreza, la exclusión social y la destrucción ambiental. Si seguimos
la ruta que señalan las principales tendencias actuales, lo único que
cabría esperar sería más de lo mismo, esto es, incremento del nú-
mero de pobres, profundización de las desigualdades sociales y ma-
yor deterioro del medio ambiente. Por tal motivo es necesario es-
tudiar las experiencias de desarrollo alternativo. Sin embargo, la enor-
me trascendencia de las experiencias exitosas del combate contra la
pobreza no se logrará entender a cabalidad, mientras sigamos cre-
yendo que la globalización es una posible solución a nuestros múl-
tiples problemas y no causa de la profundización de los mismos.

\section{Introducción}

Nuestra preocupación principal, desde que tenemos memoria, siempre ha sido cómo enfrentar la injusticia. Posteriormente, cuando comprendimos la esencia del sistema capitalista nos planteamos, ¿qué hacer para acabar con la explotación, la sobreexplotación y la expoliación?, fenómenos que se traducen en hechos empíricos, como la pobreza y la marginación social.

Hasta hace algunos años, el énfasis recaía en la búsqueda de soluciones radicales, como el cam- bio en el sistema, ya que resultaba obvio que no era posible acabar con la explotación sin dar paso a nuevas relaciones sociales de producción. En la actualidad, ante el fiero incremento de la sobreexplotación y la expoliación, así como del derrumbe del socialismo histórico, resulta poco práctico - políticamente hablando- ocuparse de la eliminación de la explotación, ya que millones de personas en el mundo preferirian ser explotadas que marginadas del sistema. Por otra parte, no existen condiciones subjetivas que animen a trabajar por la construcción de una nueva organización social diferente a la ca- 
pitalista. Adicionalmente, al haberse incrementado los niveles de sobreexplotación y expoliación, así como el desempleo y la pobreza en el mundo, resulta más urgente ocuparse de esta problemática que de la anterior.

Sin embargo, debemos estar claros. No se trata de claudicar ante el sistema; no se trata de aceptar que hemos llegado al fin de la historia, sino tan sólo de hacer una pausa. Por ahora es preciso ocuparnos de tareas más urgentes, pero sin perder en ningún momento la perspectiva; en el horizonte siempre estará marcada la necesidad de cambiar el sistema. Tal necesidad se comprenderá mejor cuando estudiemos las tendencias que presenta el mundo en la actualidad, así como sus principales efectos o consecuencias en términos socioeconómicos. Siendo achacables, tanto las tendencias como sus efectos, a la lógica y a los dinamismos propios del modo de producción capitalista.

Desde hace algunos años hemos venido ocupándonos, preferentemente, de estudiar todas aquellas experiencias de desarrollo alternativo, en tanto que soluciones parciales a la pobreza y a la marginación social. Desde inicios de la década de los noventa, nos dimos cuenta de que los pobres organizados son capaces de emprender proyectos económicos que les permiten enfrentar sus problemas de pobreza y marginación social. En línea con lo anterior, hemos publicado varios estudios donde fundamentamos teórica y empíricamente, lo que hemos dado en llamar nueva economía popular. Ciertamente, con gran sorpresa y agrado, nos enteramos que el fenómeno no es exclusivo de nuestro país, sino que también existe y ha sido estudiado y sistematizado en otros países de América Latina.

En la actualidad, ante los efectos devastadores de la globalización, se torna más importante y urgente ahondar en esta línea de investigación, razón por la cual andamos en busca de financiamiento para trabajar en lo que englobamos en el tema: experiencias exitosas de combate a la pobreza. A causa de nuestras investigaciones anteriores sabemos que existen, de allí que consideramos de suma importancia investigarlas a profundidad, a fin de lograr desentrañar cómo lo han conseguido aquellos que han superado la pobreza o están en condiciones para lograrlo y, obviamente, proceder a divulgar tales experiencias exitosas para que puedan servir a otras personas, que aún sufren la desgracia de ser pobres.

La enorme trascendencia de las experiencias exitosas del combate contra la pobreza no se logrará entender a cabalidad, mientras no tengamos una visión global de la realidad nacional y mundial; mientras sigamos creyendo que la globalización es una posible solución a nuestros múltiples problemas y no causa de la profundización de los mismos. Para ello es preciso, primero, ocuparnos de estudiar cuáles son las tendencias que presenta el mundo capitalista en la actualidad. Luego, en un segundo momento, proceder a develar las múltiples consecuencias de tales tendencias. Todo ello servirá de marco para nuestra investigación sobre las experiencias exitosas de combate a la pobreza, lo cual seguirá a nivel de proyecto, mientras no obtengamos los recursos financieros imprescindibles para realizar una investigación de campo como la proyectada. Este es el auténtico "círculo vicioso de la pobreza".

Para comprender la problemática actual del mundo, así como los límites y las posibilidades de solución, es preciso conocer cuáles son las principales tendencias que están delineando el futuro previsible. Hoy más que nunca estamos viviendo el inicio de una fase de transición hacia una sociedad mundial desgarrada por la pobreza, la exclusión social y la destrucción ambiental. Si seguimos la ruta que señalan las principales tendencias actuales, lo único que cabría esperar sería más de lo mismo, esto es, incremento del número de pobres, profundización de las desigualdades sociales y mayor deterioro del medio ambiente. En la actualidad, escribir sobre la problemática del mundo y hacerlo desde el tercer mundo, ciertamente, obliga a fruncir el ceño y a mantener una actitud lúgubre, tétrica, con olor a cementerio.

Mientras en el primer mundo se habla de la nueva economía, de la tercera vía, del crecimiento económico, de los avances tecnológicos, en el tercer mundo o submundo capitalista seguimos padeciendo casi los mismos problemas que ya teníamos hace medio siglo, aunque ahora afectan a un mayor número de personas. ¿Cuándo podremos, en América Latina, realizar algo semejante a lo efectuado en China que, en veinte años (1978-1998), redujo el número de pobres de 260 a 42 millones?'. El caso de China es de suma importancia

1. Informe del PNUD sobre la pobreza del año 2000. 
tenerlo presente, al menos por dos razones. La primera porque representa una quinta parte de la población mundial y, la segunda, porque es una sociedad que no está organizada sobre la base de la lógica del sistema capitalista, esto es, atendiendo en esencia a la búsqueda privada de ganancias y a los designios del mercado, exclusivamente. Por otra parte, durante el primer semestre del 2000, China tuvo una tasa de crecimiento del 8 por ciento, hecho que supera en mucho a los grandes bloques comerciales de occidente: a la Unión Europea y a los integrantes del Tratado de Libre Comercio en América del Norte, ya no se diga a Japón. O para ser más precisos, los países integrantes de la OCDE, que en promedio tuvieron una tasa de crecimiento del producto interno bruto de tan sólo el 4.0 por ciento ${ }^{2}$.

\section{Principales tendencias del capital en la ac- tualidad}

Pero bien, ocupémonos ahora de esas grandes tendencias que presenta la economía mundial, a fin de entender qué podrían esperar nuestros pueblos pobres y subdesarrollados.

\subsection{La globalización del capital}

La principal tendencia que domina el ámbito capitalista es la llamada globalización, la cual ciertamente está ocurriendo, pero no con toda la novedad ni con las ventajas que a menudo se piensa. Visto desde el punto de vista histórico, el fenómeno de la globalización se corresponde con la cuarta internacionalización del capital. En consecuencia, que el capital se globalice no es un fenómeno radicalmente nuevo, aunque sí posee algunos elementos novedosos que permiten diferenciarlo de los procesos anteriores.

En la primera globalización, al capital industrial le correspondió un lugar central; en la segunda le correspondió al capital comercial y en la tercera, al capital financiero". Hoy en día, el capital como un todo es el que se globaliza, ya sea bajo su modalidad de capital industrial, comercial o financiero. Precisamente es lo que observamos cuan- do, como fruto de los avances tecnológicos, los procesos de trabajo se fragmentan y deslocalizan. El capital industrial ubica parte de sus procesos productivos donde puede operar con menores costos y mayores beneficios. Las mercancías producidas en el sịstema capitalista inundan el mundo y existe una fuerte tendencia a uniformizar los gustos, lo cual permitirá una mayor estandarización de los productos. Todo ello, gracias a la penetración mundial de los medios masivos, como la televisión y el uso, ya bastante significativo, de internet.

De manera semejante, los medios electrónicos de comunicación están favoreciendo tanto el desplazamiento mundial del capital financiero como las modalidades de inversión, muy rentables para los inversionistas, pera poco beneficiosas para el bienestar de la población mundial al no destinarse a actividades propias de la economía real, sino, más bien, al ámbito monetario o al bursátil donde, si bien se amasan grandes fortunas, no se diferencia en mucho de la forma en que se gana dinero en los casinos de Las Vegas.

Ahora bien, la comprensión de la globalización resulta insuficiente si no hacemos referencia a que los sujetos de la misma no lo son el capital genérico ni capitales cualesquiera, se trata del capital personificado en lo que se denomina: empresas transnacionales, máxima expresión de la concentración y centralización del capital a nivel mundial. Hay que tener presente que las veintitrés transnacionales más importantes del mundo controlan el 70 por ciento del comercio mundial. Siendo esto así y conociendo que tales empresas manejan activos y presupuestos superiores a los de muchos estados nacionales ${ }^{4}$, resulta sobremanera ingenuo pensar que se le puede sacar algún provecho a la globalización, o que está en nuestras manos el hacerlo o que como resultado de la globalización se podrá sacar de la miseria a nuestros subdesarrollados países, como parecen creerlo gobernantes y empresarios. Si en algún momento nos integramos a la red mundial del capital, ciertamente, no será para beneficio de nuestros países, sino de las transnacionales y con enormes costos sociales y ecológicos para nosotros. Pero el fenómeno globa-

2. El País Digital, 31 de mayo de 2000.

3. Para mayores detalles sobre lo anterior, consúltese el siguiente artículo del autor: "Globalización... ¿Y nada más?", ECA. abril, 1996.

4. Se estima que las 500 empresas transnacionales más grandes controlan el 45 por ciento del producto bruto mundial, Fortune. 
lizador está posibilitando, a su vez, ampliar el poder de las empresas transnacionales, vía concentración y centralización del capital.

\subsection{Concentración y centralización del capital}

Ahora bien, el fenómeno de la globalización ha empujado a las grandes empresas a las fusiones y a las absorciones con fines muy claros: disminuir costos, propiciar megadespidos de trabajadores ${ }^{5}$; controlar merca$\operatorname{dos}^{\mathrm{n}}$; desplegarse por el mundo entero; ser más competitivas, etc. Así, a nivel internacional se informan numerosas megafusiones en el campo del petróleo, la banca, los automóviles, las comunica-
... la comprensión de la globalización resulta insuficiente si no hacemos referencia a que los sujetos de la misma no lo son el capital genérico ni capitales cualesquiera, se trata del capital personificado en lo que se denomina: empresas transnacionales, máxima expresión de la concentración y centralización del capital a nivel mundial.
Las fusiones y absorciones no sólo generan más poder económico, sino que confieren otro tipo de poderes. Por ejemplo, en Estados Unidos existen tres grandes cadenas de televisión $y$, en los últimos diez años, cada una se ha fusionado a alestá asociada a Westinghouse, que fabrica armamento militar, y se ha mostrado muy interesada en mostrar imágenes de muertos en Irak; $A B C$ pertenece a $D i s$ ney, por lo que cualquier cuestión relativa al imperialismo cultural no va a salir jamás en los informativos de $\mathrm{Mic}$ key Mouse. La propietaria de $C B S$ es General Electric, una de las compañías que más ciones, los supermercados, las bebidas, los alimentos, etc. ${ }^{7}$. Igual fenómeno ocurre en los espacios nacionales, aunque acá se realicen más con propósitos defensivos que ofensivos, aunque la resultante sea casi siempre la misma: despidos masivos de trabajadores, a fin de disminuir costos e incrementar beneficios. puestos de trabajo se han llevado desde Estados con los sindicatos para mantener los salarios a un nivel bajísimo; de modo que en esa cadena no se habla nunca del Tratado de Libre Comercios. Philip Morris, empresa parcialmente responsable del cáncer de los fumadores en el mundo, ahora Unidos a México y que allí ha roto los acuerdos

5. Algunos ejemplos de megadespidos: "Britis Aeroespace elimina 3800 empleos en el Reino Unido por la compra de Marconi Electronic Systems", El País, 16 de junio de 2000. La posible fusión del Deutsche Bank con Dresdner Bank vaticinaba 16000 despidos. La compañía Nissan, que pasó a ser controlada por Renault, suprimirá 10000 empleos. Sumitomo y Sakura al fusionarse despedirán 9300 empleados, etc. El País Digital, distintas fechas.

6. Ante la presencia en el Reino Unido y Alemania de Wall-Mart, líder mundial en supermercados, se fusionan en España Pryca y Continente y pasan a controlar 114 hipermercados, 200 supermercados y 2000 tiendas de descuento, lo cual implica que sus casas matrices francesas Promodés y Carrefour, ya están fusionadas. Lo mismo podría ocurrir con el alemán Metro y el holandes Ahold. El País Digital, distintas fechas.

7. Algunos ejemplos de fusiones: Chevron y Texaco; Motorola compra General Instrument por 11000 millones de dólares; Viacom compra CBS en 37000 millones de dólares para crear el segundo grupo mundial de comunicación; la hotelera Hilton compra Promus en 4000 millones de dólares y se constituye en la séptima cadena hotelera del mundo con 1700 hoteles, muy lejos por cierto de Cendant Co, que controla 6315 hoteles y 642630 habitaciones; Vodafone Airtouch (30 millones de usuarios de telefonía móvil) busca fusionarse con Bell Atlantic (40 millones de clientes de telefonía local); Air France y Delta Airlines se unen para crear la tercera alianza mundial, las dos anteriores son Oneworld, que integra a British Airways, American Airlines, Iberia y Catay Pacific, y la otra es Star Airlines, que engloba a Lufthansa, United Airlines, Air Canada, Thai Airways Internacional y Scandinavian Airlines; Sumitomo y Sakura, dos bancos japoneses, se fusionan y crean el segundo banco del mundo, luego de la fusión resultante de Dai Ichi-Kangyo-Fuji-IBJ, que ocupa el primer lugar; DASA y Aérospaciale al fusionarse ocuparán el primer lugar en Europa y el tercer puesto a nivel mundial, sólo después de las estadunidenses Boeing y Lockheed-Martin; Volvo y Mitsubishi (camiones y autobuses), Peugeot-Citren y Ford se alían para fabricar motores diesel; la sudafricana SAP y Nomura compran Pilsner Urquell, la primera cerveza checa. El País Digital, distintas fechas.

8. El País Semanal, 30 de abril de 2000. 
adquiere Nabisco Holdings, el mayor fabricante de galletas, que ya antes había adquirido Kraft; ambas empresas productoras de alimentos serán operadas ahora con la ética de las tabacaleras y, ciertamente, no augura mucha salud a sus clientes ${ }^{9}$.

Otro elemento importante que debe tenerse presente es cómo se articulan los capitales y, en consecuencia, sus intereses. Un ejemplo interesante es el caso del Deutsche Bank, que adquiere al Banker Trust y se ubica entre los primeros tres grandes bancos del mundo; pero sus intereses están también en la transnacional Daimler Chrysler -de la cual posee el 12 por ciento de sus acciones-, en la aseguradora Allienz —con el 4.8 por ciento de sus acciones-, en la firma de seguros y reaseguros Munchener Ruckversicherungs —con el 8.0 por ciento-, así como en la cementera Heildlberg Zement y en las compañías metalúrgicas Linde y Metallgesellschaft ${ }^{\prime \prime}$.

En la industria automovilística, como claro ejemplo de la tendencia mundial el resultado de las fusiones y absorciones, el control del mercado mundial la tienen tres o cuatro grandes empresas transnacionales, de origen estadunidense, alemán y japonés".

Si bien la concentración y centralización del capital son características del sistema capitalista, las magnitudes que alcanzan estos procesos, en la actualidad, hacen de los mismos fenómenos inéditos, cuyas consecuencias son imprevisibles en su totalidad. Sin embargo, parece claro que el control que tendrán sobre los mercados será omnímodo, de lo cual cabe esperar prácticas monopólicas u oligopólicas, que redundará en serios prejuicios para los consumidores. Tal tendencia se intenta corregir o al menos aliviar mediante la promulgación de leyes a favor de la libre competencia. No obstante, siempre queda la interrogante, ¿podrán los estados nacionales promover la competencia cuando la economía mundial está pasando a ser controlada por unas cuantas empresas transnacionales, al menos en aquellas actividades más importantes, como son la industria automovilística, las comunicaciones, los energéticos, las finanzas, los medicamentos, los alimentos e, inclusive, los supermercados? Por ejemplo, en nuestro país, las administradoras de los fondos de pensiones se han fusionado porque sus respectivas casas matrices lo han hecho pero, ¿qué diferencia existiría si se mantuvieran como empresas independientes cuando responden a los mismos intereses? Eso es lo que actualmente vivimos en la compra de combustibles petrolíferas, ¿variaría la situación si existiera una ley de libre competencia? ¿Quién y cómo la haría cumplir?

\subsection{Elevación de la composición orgánica del capital}

La otra característica que presentan las empresas, hoy en día, es su tendencia a elevar la composición orgánica del capital; esto es lo mismo que decir la relación capital/trabajo por empresa. Gracias a las innovaciones tecnológicas - la mecanización, automatización, robótica y computación-, las empresas destinan cada vez una menor proporción de su capital a la contratación de trabajadores y una mayor proporción a los factores productivos materiales, como maquinaria, equipo, materias primas, etc. Ello se traduce en un incremento en la productividad del trabajo de los pocos trabajadores

9. El País Digital, 26 de junio de 2000.

10. El País Digital.

11. Daimler Crysler busca adquirir el 33.4 por ciento de la Mitsubishi Motors y pasaría a ser la tercera lirma en importancia en materia automovilística, sólo detrás de General Motors y Ford, pero por sobre Toyota que ha sido relegada al cuarto lugar. General Motors ya controla en Japón a Isuzu, Hino y participa en Suzuki; también posee acciones de la italiana Fiat. Busca adquirir a la coreana Daewood. Ford controla el 33 por ciento de Mazda. Renault, por su parte, se ha vinculado a Nissan, de la que posee el 36.8 por ciento. El País Digital, distintas fechas. 


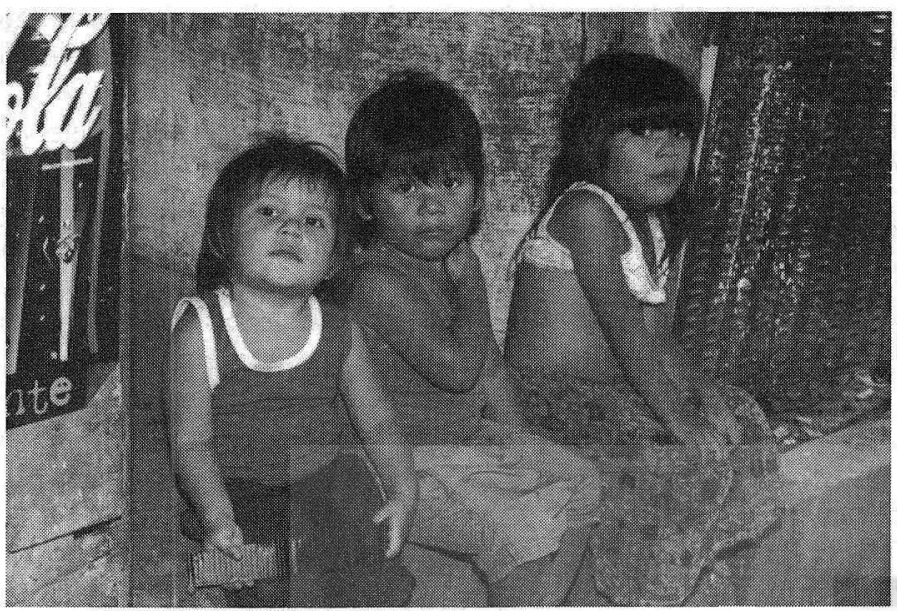

cual se traduce en 147.4 millones de dólares por cada empleado; o Fortis, cuyos activos ascienden a 407043.2 y tiene 62000 empleados, lo que da una relación de 6.56 millones de dólares por cada trabajador ${ }^{12}$. ¿Se imagina las enormes cantidades de capital que se requerirían para resolver el desempleo en el mundo?

Seguramente si David Ricardo ${ }^{13}$ conociera hasta dónde ha llegado la elevación de la composición orgánica del capital, quizá sería un férreo anticapitalista. David Ricardo, en su obra Principios de economía política, sostenía:

Creía yo que la clase trabajadora se be-

contratados, que posibilita a las grandes empresas aumentar sus beneficios, ya que al disminuir los niveles de competitividad, los incrementos en la productividad no se traducen en la disminución de los precios ni en incrementos salariales, a pesar de la situación de desempleo estructural que predomina en el mundo.

Además del incremento en la relación capital y trabajo señalada antes, la magnitud de las inversiones alcanza tales cifras, que las posibilidades de crear nuevas empresas que disputen el mercado a las existentes se va convirtiendo en una vía muerta. Es obvio que muchas empresas nacionales, consideradas grandes en nuestros mínimos mercados, no son capaces de competir ni siquiera con las empresas extranjeras medianas de origen mexicano, ya no digamos con las monstruosas empresas transnacionales.

Algunos ejemplos que nos permiten comprender lo elevada que es la relación capital y trabajo los encontramos al vincular los activos con el número de empleados de las empresas más grandes. Así, tenemos que el Deutsche Bank cuenta con aclivos cuyo valor asciende a 841796.9 millones de dólares y sólo tiene contratados a 93232 empleados, lo que da una relación de 9 millones de dólares por cada empleado; o bien, la corporación Fannie Mae, cuyos activos ascienden a 575167.4 y tiene contratados a tan sólo 3900 empleados, lo neficiaba igualmente con el uso de la maquinaria [...] Como entonces me parecía que habría la misma demanda de trabajo que antes, y que los salarios no serían más bajos, pensaba que la clase trabajadora participaría, al igual que las otras clases sociales, del provecho de la baratura general de las mercancías, causada por el uso de maquinaria.

Estas eran mis opiniones, que continúan incólumes en lo que se refiere al terrateniente y al capitalista; pero estoy convencido ahora de que la sustitución del trabajo humano por la maquinaria es, a menudo, muy perjudicial a los intereses de la clase trabajadora.

\subsection{La conformación de megamercados o gran- des bloques comerciales}

Quizá el mejor ejemplo de lo anterior es la creación del acuerdo de libre comercio entre Canadá, Estados Unidos y México, al cual están buscando adherirse los países centroamericanos. Pero también la Unión Europea, aunque con una profundidad mayor, ha constituido un solo mercado gigantesco. En Suramérica encontramos el Mercosur, el Pacto Andino, etc. Lo interesante o, mejor dicho, lo lamentable de estos acuerdos es que se facilita la movilidad del capital, bajo cualesquiera de sus modalidades, pero siempre permanece restringida la movilidad de las personas, hecho

12. Fortune, julio de 2000.

13. Cuyas obras deberían de estudiar en serio nuestros economistas, dado que Marx les asusta tanto, seguramente aprenderían mucho sobre economía y honestidad intelectual. 
que pone en evidencia la finalidad que se persigue o a qué intereses responden los mismos.

La verdad de las cosas es que el mundo se ha convertido en un solo mercado para las grandes transnacionales. Así, durante 1999, las nueve empresas más importantes del planeta tuvieron ingresos de 1293.4 miles de millones de dólares y ganancias por un valor de 47579.7 millones de dólares. De estas nueve empresas, una es alemana (Daimler-Chrysler), tres son japonesas (Mitsui, Mitsubishi y Toyota) y el resto, estadunidenses (General Motors, Wal-Mart Stores, Exxon Mobil, Ford y General Electric); a esta última empresa le corresponde el 22.5 por ciento del total de las ganancias que tuvieron las nueve empresas.

En el caso de la región centroamericana, es común señalar quę nuestros países no tienen viabilidad futura desunidos, que la integración centroamericana es una necesidad histórica. Sin embargo, nunca hemos pasado de conformar meras uniones aduaneras, de las cuales los beneficiados no han sido los trabajadores de la región, sino los grandes empresarios; sin embargo, persistimos en lo mismo y presentamos los mismos argumentos: que se generarán cientos de miles de nuevos puestos de trabajo. No obstante, las cifras absolutas de desempleados en la región y en el mundo siguen incrementándose.

\subsection{La flexibilización laboral}

Esta tendencia - que básicamente consiste en eliminar las conquistas laborales que los trabajadores ganaron después de muchos años de lucha que costó sangre-, es la alternativa que encuentra el capital ante la tendencia mundial de incrementarse el número de desocupados. Pareciera que es preferible tener un empleo, aun de características precarias, a no tenerlo, pese a que de esa manera incrementan sus beneficios los empresarios capitalistas.

Mediante la flexibilización laboral se busca despedir a los trabajadores sin indemnización, contratarlos por horas, flexibilizar los salarios, eliminar los contratos colectivos y disminuir cuando no eliminar las prestaciones sociales. El salario directo, la cantidad en efectivo que recibe el trabajador por quincena o por mes, se busca reducir; el salario indirecto, o sea las prestaciones sociales, se busca disminuir o eliminar y lo mismo ocurre con el salario diferido, el seguro por vejez, invalidez o muerte, el cual se quiere cargar al salario directo del trabajador.

Una muestra rápida de las consecuencias de la flexibilización laboral la encontramos en Argentina, donde

De cada cien personas incorporadas a la vida laboral, 13 están totalmente desocupadas; doce trabajan en forma intermitente; veinte tienen un empleo regular informal y doce son trabajadores autónomos carentes de seguridad social. Sólo el 40 por ciento de la población económicamente activa tiene un empleo estable en blanco, pero uno de cada tres, trabaja jornadas superiores a la legal sin recibir compensación alguna en cerca de la mitad de los casos ${ }^{14}$.

Este proceso de flexibilización laboral se está aprovechando de los bajos niveles de organización sindical de los trabajadores, en esta fase de baja marea laboral. Desafortunadamente, el impacto social será de fatales consecuencias para la vida y la dignidad de los trabajadores; ya no hay quien dude de la precarización del trabajo.

Segǔn la Organización Internacional del Trabajo, en los diez años últimos, la proporción de trabajadores sindicados respecto a la fuerza de trabajo total ha disminuido, a veces sensiblemente, en casi todos los países. Para medir lo anterior, dicha Organización utiliza el índice de sindicación. Pues bien, en 1995, ese índice se redujo, en Estados Unidos, 21.1 por ciento; en Japón, 16.7 por ciento; en Australia, 29.6 por ciento; en Alemania, 17.6 por ciento; en Francia, 37.2 por ciento; y en el Reino Unido, 27.7 por ciento ${ }^{15}$.

14. Organización Internacional del Trabajo (OIT), La flexibilización laboral en Argentina.

15. Oficina Internacional del Trabajo, El Trabajo en el mundo 1997-98. 
Pero, ¿cuál es la justificación para la flexibilización laboral? Existen muchos argumentos, pero encontramos uno que resultará muy útil a nuestros empresarios.

Quienes desde Washington -Fondo Monetario Internacional y Banco Mundial- promueven la liberación del mercado laboral sostienen que las leyes y regulaciones establecidas para proteger a los empleados sólo sirven para impedir la reestructuración industrial, que es prerrequisito para un dinámico crecimiento económico... ${ }^{16}$.

Y para presentar un ejemplo de cómo los gobiernos asumen la flexibilización laboral, veamos el caso colombiano (adicionalmente mencionamos que entrenta un 20 por ciento de desempleo).

Esta iniciativa gubernamental marca una clara tendencia: ya nadie tendrá un empleo vitalicio; las nóminas de las empresas serán cada vez más delgadas y, en cambio, contarán con ejércitos de proveedores de servicios; se remunerará la labor cumplida; cada vez más el aseguramiento en seguridad social correrá por cuenta del trabajador; se impondrá el trabajo por horas y por jornadas parciales; despedir a un trabajador será tan fácil como contratarlo... ${ }^{17}$

Parece claro que la flexibilización laboral, como suele ocurrir en el capitalismo, es un mecanismo de la clase capitalista para disminuir costos a costa de la clase trabajadora. Si esto no es lucha de clases, al menos es una agresión de la clase capitalista a la clase obrera.

\subsection{La incorporación de la mujer al trabajo} asalariado, nueva modalidad de explotación

En nuestros días, las maquilas constituyen una de las pocas oportunidades que existen en el tercer mundo para tener acceso a un empleo; pero se nu- tren de trabajo femenino mal pagado y en condiciones precarias. El nuevo paisaje urbano está constituido por miles de mujeres jóvenes, de extracción campesina, que se dirigen a las fábricas al amanecer. Un ejemplo de lo anterior es Bangladesh: según datos de Naciones Unidas, en 1978 sólo había 4 fábricas de prendas de vestir; en 1995 ya habían 2400 y daban empleo a 1.2 millones de trabajadores. El 99 por ciento eran mujeres menores de 25 años.

Los maquiladores del país, en muchos casos, obligan a las mujeres a trabajar más horas de las normales, sin pagarles las horas extras; se les häcen los descuentos del Instituto Salvadoreño del Seguro Social, pero no se hace el pago correspondiente; si una mujer sale embarazada se despide. Además, existe una elevada tasa de rotación femenina, a fin de aprovechar la energía de las mujeres más jóvenes ${ }^{18}$.

También abundan las noticias sobre las fábricas que cierran y dejan en la calle a las obreras, sin cancelarles ningún tipo de indemnización. Ciertamente, la proporción de mujeres que se incorporan a la actividad laboral está aumentando en el país y en el mundo, pero la media salarial de las mismas todavía permanece por debajo de la media salarial de los hombres. Todo ello se traduce en mayores beneficios para los empresarios capitalistas.

Se podría pensar que lo anterior es sólo un problema del tercer mundo; sin embargo, estudios de Eurostat (Oficina Estadística de las Comunidades Europeas) revelan que las mujeres ganan, en la Unión Europea, un 28 por ciento menos que los hombres y adicionalmente se establece que el 80 por ciento de los empleos de tiempo parcial están ocupados por mujeres. En la Unión Europea, el 15 por ciento de los trabajadores tienen salarios bajos, esto es, inferior al 60 por ciento del salario medio nacional. De los 16.5 millones de trabajadores que

16. WWW.seguridadsocial.com.co/jul99a.htm. Se mueve la butaca.

17. Ibid.

18. Sobre el particular, consúltese el trabajo de J. Arriola y A. Candray, Derechos prohibidos, negociación colectiva y sindicatos en El Salvador. Documento de investigación, UCA, 1994. 
tienen un salario bajo, el 77 por ciento son mujeres. La situación es peor en Austria (cuyo porcentaje es del 86 por ciento), Reino Unido y Holanda ( 81 por ciento), y Alemania ( 80 por ciento) ${ }^{19}$.

\subsection{Privatización de activos, servicios públicos y bienes públicos}

Como ya lo hemos manifestado en numerosas ocasiones, el único objetivo que persiguen estas medidas es ampliar los espacios de valorización del capital. O si se prefiere, abrir espacios a la inversión privada, algunos ya viejos, otros novísimos. Así, en todo el mundo se han privatizado no sólo empresas estatales productoras de bienes y servicios, que normalmente son propiedad de empresarios capitalistas, como los bancos, ingenios azucareros, beneficios de café, hoteles, etc., sino también empresas de servicios públicos que tradicionalmente han sido propiedad estatal, como las telecomunicaciones, la generación y distribución de energía eléctrica y, últimamente, se está caminando hacia la privatización de la captación y distribución de agua ${ }^{20}$ o de los servicios gratuitos prestados por el Estado, como los de salud y la educación. El argumento esgrimido, o la justificación para estos cambios, ha sido la supuesta eficiencia del mercado, lo cual -aunque muy cuestionable - podría justificarse si todas las personas contaran con ingresos suficientes para comprar tales servicios, pero resulta por completo inadmisible cuando se trata de pueblos pobres, que acceden con grandes dificultades a los servicios de salud y educación, pese a que estos sean gratuitos. El caso del agua es mucho más grave, ya que sin aire y sin agua se pierde la vida con mayor celeridad que sin alimentos.

\subsection{Las megaganancias empresariales}

Una tendencia resultante de lo anterior es que las megaempresas obtienen megaganancias. Si el capital se concentra y centraliza, si se amplían los mercados, si se eleva la composición orgánica del capital, si se dan prácticas monopólicas u oligopólicas, elc., es obvio que la magnitud de las ganancias liene que crecer. Por ejemplo, algunas mega-

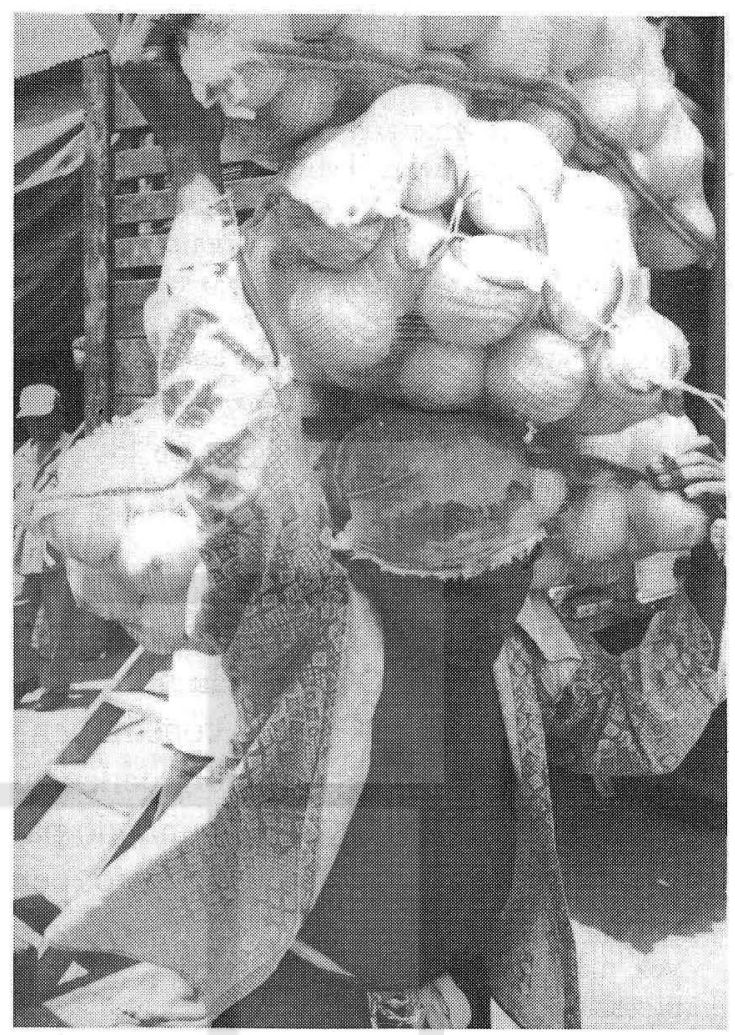

ganancias obtenidas por algunas grandes empresas, en 1999, fueron las siguientes: General Electric obtuvo 10717.0 millones de dólares; Citigroup, 9 867.0; Royal Dutch/Shell Goup, 8 584.0; Ford Motor obtuvo 7237.0 millones de dólares; General Motors, 6002.0 millones de dólares ${ }^{21}$. Y durante el segundo trimestre del 2000: Coca Cola informa haber ganado 926 millones de dólares; $\mathrm{Ci}$ tigroup, 3000 millones; Chase Manhattan, 1090 millones; Boeing, 654 millones, etc. Estos datos revelan la irracionalidad de la racionalidad capitalista, ya que uno se pregunta, ¿qué sentido podrá tener el obtener tales beneficios, mientras la mitad de la humanidad vive en la pobreza?

Siendo así las cosas, no resulta extraño que la riqueza de las tres personas más ricas del mundo sea superior al producto interno bruto de 48 naciones con 600 millones de personas ${ }^{22}$, lo cual, cierta-

19. El País Digital, 8 de agosto de 2000.

20. Se pretende fijar el precio del agua según el coste total de recuperación; esto se planteó en el II Foro Mundial del Agua, celebrado en La Haya, en marzo del 2000. De acuerdo con ello, los pobres del mundo están condenados a morir de hambre y sed.

21. Fortune Global 500.

22. El País, 11 de abril de 2000. 
mente, es el colmo de la irracionalidad del sistema capitalista.

Pero bien, presentemos alguna información sobre los efectos que tienen las tendencias anteriores y tratemos de reflexionar sobre sus consecuencias futuras para la vida del planeta y sus seres vivos, en particular para las personas.

\section{Los efectos de las tendencias actuales sobre las personas}

Una apretada síntesis de algunos de los efectos, de los cuales nos ocuparemos, la presenta Sergio Ramírez:

Una red de cincuenta organizaciones agrupadas en el Turning Point Project prevenía en The New York Times, hace algún tiempo, sobre los riesgos ya visibles de "la monocultura global". Ese fenómeno arrasa cada vez más con los empresarios locales que, al verse compelidos a vender sus empresas a los grandes consorcios globales, pasan a ser rentistas; liquida la producción de alimentos que resulta más barato importar; empobrece el medio ambiente al destruir los bosques y contaminar las fuentes de agua, en Brasil o en Canadá, y arrebata a las naciones sus rasgos de identidad fundamentales. Disney puede uniformar las conciencias, y McDonald's, los gustos ${ }^{23}$.

Un primer dato que resulta realmente alarmante, por ser un sin sentido en términos de posibilidades materiales, es el hecho de que según la FAO, 800 millones de personas pasan hambre en el mundo, que equivale a un número de personas superior al que hay en Europa y Estados Unidos. $Y$ decimos que es un sin sentido en términos de posibilidades materiales, ya que con el desarrollo alcanzado en la actualidad, existe suficiente capacidad material para satisfacer las necesidades de alimentación de la totalidad de las personas que habitamos el planeta. Pero si 800 millones de indi- viduos padecen hambre, es porque no pueden comprar los alimentos, y si no pueden comprarlos es porque el sistema es incapaz de generar ingresos para todas las personas, lo cual lo convierte en un sistema irracional y a su vez cruel, debido a que mientras unos cuantos acumulan innecesariamente riqueza, otros padecen hambre.

Según Naciones Unidas, 80 millones de personas carecen por completo de servicios de salud, 849 millones padecen de desnutrición, incluidos ahora algunos habitantes de la ex Unión de Repúblicas Socialistas Soviéticas. Más de 260 millones no pueden ir a la escuela. ¿Qué sentido puede tener el desarrollo en el capitalismo, si no genera bienestar para las personas?

Según el último informe del Programa de las Naciones Unidas para el Desarrollo (PNUD), 1200 millones de personas sobreviven con menos de un dólar al día en el mundo, lo cual equivale a alrededor de una quinta parte de la población mundial. Pero si incluimos a quienes perciben menos de dos dólares, la magnitud se incrementa a 3000 millones de personas, esto es, la mitad de la población mundial.

Según el Banco Mundial, en Indicadores del Crecimiento Mundial 2000, una sexta parte de la población mundial acapara el 80 por ciento de la renta mundial. Esto implica que sólo el 20 por ciento de la renta está destinada a cinco sextos de la población mundial, mientras que al 20 por ciento más pobre apenas le corresponde el 1.6 por ciento. En América Latina, según el Banco Interamericano de Desarrollo, el 5 por ciento de la población se apropia de una cuarta parte de la riqueza, o sea, del 25 por ciento. ¿Podrá alguien, en su sano juicio, negar que la riqueza de unos pocos es a costa de la mayoría?

En el II Foro Mundial del Agua, celebrado en marzo de este año, se reconoció que mas de 1400 millones de seres humanos (alrededor de la cuarta parte de la población mundial) no tienen acceso al

23. Sergio Ramírez, "El callejón más oscuro", El País, 22 de agosto de 2000. 
agua potable. $\mathrm{Y}$ hay quienes piensan que privatizando el servicio se mejorará el acceso al mismo, ¿cómo es posible tanta irracionalidad?

En los países desarrollados, esto es, en aquellos que conforman la OCDE - pese a que, en promedio, la OCDE registra una tasa de crecimiento del producto interno bruto de un 4.0 por ciento-, los porcentajes de desempleo son elevados: en Estados Unidos es del 4.0 por ciento; en Japón, del 4.8 por ciento; en España, del 14.1 por ciento; en Alemania, del 8.5 por ciento; en Francia, del 9.8 por ciento; en Italia, del 11.0 por ciento; en Reino Unido, del 5.7 por ciento; en Canadá, del 6.8 por ciento; en la Unión Europea, del 8.5 por ciento; en la zona euro, del 9.2 por ciento, y en la OCDE, del 6.3 por ciento ${ }^{24}$. En 1996, el número de desempleados en la OCDE ascendía a los 36 millones de personas. Y tengamos presente que las cifras de desocupados sólo incluyen a quienes persisten en buscar empleo, por lo que la cifra de parados, obviamente, es mayor. Así, por ejemplo, España, que tiene una tasa de desempleo de sólo 14.1 por ciento, tiene una tasa de empleo del 55 por ciento. De la población de 16 a 64 años, o sea la que está en edad de trabajar, sólo un poco más de la mitad lo hace.

Este fenómeno no es exclusivo de España, sino del mundo. Y la situación todavía es peor en países como el nuestro, donde lo que predomina es el subempleo. Según la DIGESTYC, la población económicamente activa asciende a 2403194 personas. en la industria, el descenso había sido del 10.9 por ciento $^{25}$. Y si esto ocurre en el paraíso del capitalismo, ¿qué se puede esperar en el tercer mundo?

América Latina tiene 500 millones de habitantes, de los cuales 224 millonets son pobres. De cada diez empleos, siete son inestables, según la CEPAL. Por otra parte, mientras muchos no tienen qué comer, otros son presas del consumismo descontrolado en los países del primer y tercer mundo, lo cual no sólo es un efecto de la globalización, sino que, a su vez, causa de la degradación ambiental. Respecto al fenómeno consumista, fray Beto manifiesta:

Para ellos un shopping center es un templo religioso del consumo. No se puede ir como uno quiera, hay que ir con ropa de misa dominical y usted pasa por aquellos claustros, por aquellas capillas con venerables objetos de consumo y rodeado de bellas sacerdotisas, si usted no puede comprar se siente en el infierno. Si puede comprar a crédito está en el purgatorio; pero si tuvo manera de comprar, está en el reino de los cielos... La globalización neoliberal no quiere ciudadanos quiere consumidores ${ }^{26}$.

Ocurre que el 20 por ciento más rico del globo consume el 86 por ciento de lo que se produce; mientras que al 80 por ciento restante de la población sólo le queda el 14 por ciento. Y así, ¿cómo y cuándo podremos salir de la miseria? ¡Y estas son De estas, las ocupadas son $2227471 \mathrm{y}$, según la misma fuente, la tasa de desempleo es de sólo 7.31 por ciento; sin embargo, los cotizantes al Instituto Salvadoreño del Seguro Social son tan sólo 636 mil personas. De ello se deduce que la mayoría de personas labora en actividades informales o están subempleadas, atendiendo al tiempo que laboran o a los ingresos que perciben.

El 40 por ciento de la población acliva ocupada en Estados Unidos tenía, en 1993, ingresos inferiores a los de veinte años atrás. El salario real promedio por hora en el sector servicios era 4.6 por ciento inferior al de 1973 ;

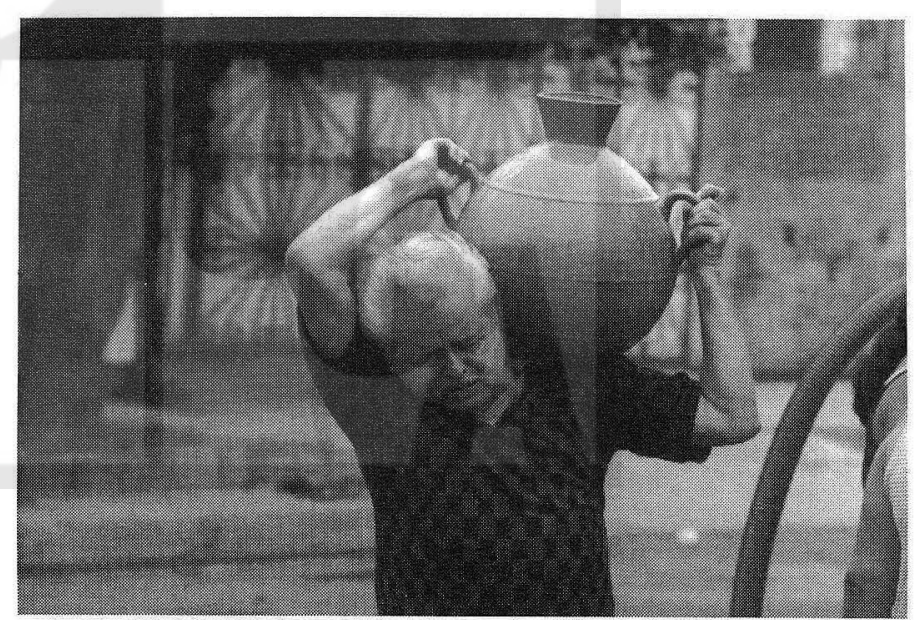

24. El País Digital, 31 de mayo de 2000.

25. J. Dalaker y M. Naifeh (1998), citado en La declinación de la Economía Global por Jorge Beinstein, ponencia presentada en el I Encuentro sobre globalización y problemas del desarrollo, La Habana, 1999.

26. Frei Beto, "Hay que organizar la esperanza", El Economista de Cuba. 
las maravillas del sistema capitalista que tantos defienden y tan pocos comprenden! $O$ sencillamente no les importa.

Más de 35000 personas mueren diariamente de hambre y malnutrición en el mundo. Y cada año mueren 7.7 millones de recién nacidos. Seguramente alguien podrá pensar que son mecanismos naturales de autorregulación de la población mundial; pero no, se trata de un fenómeno social. Tan social como el sistema en el cual vivimos y morimos de hambre. Ocurre que los antiguos países integrantes de la fenecida Unión de Repúblicas Socialistas Soviéticas han visto reducido su producto interno bruto real en alrededor del 50 por ciento, así como la esperanza de vida. Por ejemplo, en la Federación de

Rusia se observa el -5.9 , según el Informe de la Pobreza del PNUD de 1998.

Con datos como los anteriores no resulta extraño constatar que en los noventa creció, como nunca antes, la inseguridad urbana... Esa inmensa criminalidad emergente es la base social de la delincuencia organizada. Y no me refiero a El Salvador. El fenómeno que padecemos los salvadoreños es mundial y se atribuye a los efectos que han tenido los programas de ajuste estructural y la globalización. No lo digo para consolarnos, sino para que sepamos quiénes son los responsables. Y aún así, existen quienes claman por la tercera generación de reformas, dentro de la cual se incluye la

llexibilización laboral.

3. Los efectos del estilo de crecimiento actual sobre el medio ambiente

A partir del informe GEO-2000, elaborado por el Programa Medioambicntal de Naciones Uniclas, se desprende que la tendencia al deterioro de la Tierra es terrible. Veamos alguna información contenida en el mismo.
En la agricultura, por ejemplo, los efectos de la revolución verde están causando más daños que beneficios, así tenemos para el caso que el uso de fertilizantes químicos nitrogenados desde la década de los sesenta se ha quintuplicado, pasando de 12 a 80 millones de toneladas anuales y el efecto de liberar nitrógeno en el ambiente puede ser la alteración del crecimiento, así como la composición de las especies y la reducción de su diversidad.

La exposición a productos químicos peligrosos ha generado efectos negativos, desde malformaciones congénitas hasta problemas de cáncer. Sólo el uso de pesticidas provoca entre 3.5 y $5 \mathrm{mi}-$ llones de personas envenenadas cada año.
Las emisiones de dióxido de carbono se han cuadriplicado desde 1950 y es conocido el efecto invernadero que esto provoca.

El Foro de Malmoe (Suecia) celebrado en junio 2000, plantea que:

Los principales desafíos contra la naturaleza identificados en la reunión de Río de Janeiro (Brasil), en 1992, se han globalizado y agudizado por los hábitos consumistas insostenibles de occidente. La presión demográfica (la tierra tendrá 3000 millones más de habitantes dentro de 50 años) y el desarrollo de megaciudades, el tremendo riesgo de cambio climático, la crisis de agua dulce y sus consecuencias para la seguridad alimentaria, la insostenible explotación de los recursos biológicos, la sequía y la desertificación, la deforestación incontrolada, el aumento de la gravedad de los desastres naturales, las amenazas a la salud humana y el medio ambiente por la contaminación de productos químicos peligrosos son las cuestiones más urgentes ${ }^{27}$.

27. El País Digital, 31 de mayo de 2000. 
Pero eso no es todo, nos encaminamos claramente hacia un desastre ecológico si los países desarrollados no cambian su modelo industrial de crecimiento y consumismo. Ramonet ya indicaba, en 1997:

Seis millones de hectáreas de tierra cultivable desaparecen cada año por la desertificación. Los equilibrios ecológicos están fragilizados por la contaminación industrial de los países del norte ${ }^{28}$.

Entre 10 y 17 millones de hectáreas de bosque desaparecen cada año. Cada año, unos seis mil especies son borradas del planeta ${ }^{29}$.

Estados Unidos es responsable del 50 por ciento de las emisiones de gas carbónico en los países industrializados ${ }^{30}$.

\section{Los límites al desarrollo}

A mí me resulta obvio pensar que el principal obstáculo para alcanzar el desarrollo de la humanidad se encuentra en la racionalidad del sistema capitalista, la cual se reduce a la búsqueda de maximizar beneficios y acumular riqueza sin límites. $\mathrm{Y}$, en ese proceso endiablado, poco importa la destrucción que se haga de la naturaleza, de la cual se usa y abusa sin importar las consecuencias futuras. Pero lo mismo se hace con los trabajadores, quienes son los que realmente generan riqueza y a quienes se les paga o emplea lo menos posible. El sueño de los capitalistas individuales es prescindir absolutamente del costo de la energía humana y, en la medida que la automatización de los procesos de trabajo lo permiten, lo hacen, sin ninguna otra consideración que no sea la de disminuir costos. Todo ello va generando una considerable masa de personas excluidas del sistema formal de trabajo asalariado que, para sobrevivir, tienen que integrarse en las actividades informales de la economía, esto es, en formas de trabajo no capitalistas.

Si lo anterior es el obstáculo principal, qué límites concretos encontramos para negar toda posibilidad de que el sistema genere desarrollo para toda la humanidad. Para ello recordemos las ten- dencias señaladas antes. Veamos. Si el sistema permite, posibilita y exige la concentración de la riqueza, es obvio que cada vez serán más los excluidos del acceso a la misma. Si las empresas incrementan cada vez más la relación capital y trabajo, es obvio que la generación de un nuevo puesto de trabajo exigirá cada vez mayores magnitudes de inversión, lo cual supondría excesivamente altas tasas de crecimiento económico, porque - como ya veíamos- pese a que los países desarrollados tienen tasas de crecimiento aceptables, el desempleo existe y persiste y la única salida, más no la solución, que se les ocurre a los intelectuales del sistema y a sus comparsas nacionales es la flexibilización laboral, la cual precariza el trabajo asalariado. Sin embargo, el mayor crecimiento económico provocaría una mayor degradación ambiental. Basta pensar en que las mayores empresas del mundo se dedican a la producción de automotores y combustible, a partir del petróleo, y que los vehículos son los principales responsables de la emisión de bióxido de carbono, lo cual provoca el efecto invernadero, esto es, al elevarse más la temperatura del globo, se pone en peligro la vida en el planeta.

Si el sistema es incapaz de generar suficiente empleo remunerado dignamente ${ }^{31}$, sobre todo en el tercer mundo, es obvio que mientras vivamos en una economía de mercado, quienes no tengan ingresos no podrán acceder a las mercancías ni satisfacer sus necesidades $y$, en consecuencia, les resultará imposible salir del subdesarrollo. Teniendo esto en mente, debería ser obvio que la privatización de los servicios públicos, gratuitos o no, lo único que provocará en las personas es más subdesarrollo porque no podrán tener acceso a los mismos, ya sea porque tendrán que pagar por ellos o porque sus precios habrán subido, atendiendo a la racionalidad capitalista.

Otra forma sencilla de mostrar la irracional racionalidad capitalista es recordar cierta información divulgada por el Foro Global de Investigación de la Salud, presentada en Ginebra, en mayo de este año de fin de siglo.

28. Ramonet Ignacio, Le Monde Diplomatique, noviembre, 1997.

29. Ibid.

30. Ibid.

31. Recientemente leíamos que en Trinidad y Tobago, un país tercer mundista, se había instalado una fábrica dedicada a producir gas licuado, con una inversión de mil millones de dólares, pero sólo había generado 86 empleos. 
Los sectores público y privado gastan en todo el mundo cerca de 13 billones de pesetas anuales en investigación sanitaria, pero distribuyen ese dinero de un modo extremadamente ineficaz e injusto. Las enfermedades que causan el 90 por ciento de la mortalidad e incapacidad en el mundo sólo reciben el 10 por ciento del dinero dedicado a investigación médica.... ${ }^{32}$

Y uno podría preguntarse, intrigado e indignado, ¿por qué esto es así? Y la respuesta sólo puede ser una: esta es la racionalidad capitalista. Importan las enfermedades de quienes pueden pagar por los medicamentos, ¿o acaso no es ésta la lógica del mercado?

Si usted no lo cree, vea lo que a continuación señala el informe ya citado.

Tanto la industria farmacéutica como las instituciones públicas de investigación se concentran en los problemas sanitarios típicos de occidente, muchas veces ligados al envejecimiento, a la obesidad y al estilo de vida de los países desarrollados ${ }^{13}$.

Pero los límites de los que nos veníamos ocupando no han terminado. Decíamos que la globalización, impulsada por las empresas transnacionales, ha exigido la apertura comercial para competir en "igualdad de condiciones", en nuestros mercados locales, con las empresas domésticas que generaban empleo para los connacionales. Este proceso ha generado y, si nada lo altera, seguirá generando quicbras de empresas nacionales de distintos lamaños y lanzando a la calle a muchos trabajadores, para quienes la reubicación resulta casi imposible, por lo que pasarán a formar parte del ejército de desempleados o del sector informal, en condiciones precarias de vida y de trabajo, lo cual significa una profundización de la pobreza.

También existen límites financieros para salir del subdesarrollo. Mientras las megaempresas financieras como el Citygroup y el Bank of América ob- tuvieron millonarias ganancias el año pasado (9867 y 7882 millones de dólares ${ }^{34}$, respectivamente), la servidumbre de la deuda externa de los países del tercer mundo asciende a 2.5 billones de dólares; su servicio absorbe el 25 por ciento de los ingresos por exportaciones. Brasil deberá pagar en el año 2000, 52000 millones de dólares en concepto de intereses de su deuda externa e interna ${ }^{35}$.

Los límites al desarrollo también se encuentran en la profundización de la dependencia de nuestros países, no sólo en lo económico, sino también en los ámbitos social, político y cultural. Aunque ahora se le llame a esta realidad globalización $\mathrm{y}$, lejos de cuestionar la inversión extranjera -tal como lo hicieron los antiguos dependentistas-, ahora se clama por la inversión extranjera, ya que se la percibe como la gran salida a nuestra condición de subdesarrollo. Por tanto, los gobiernos tercer mundistas compiten entre sí, en busca de ofrecer las mejores condiciones a los inversionistas extranjeros, a fin de que vengan a nuestros países a sobreexplotar la fuerza de trabajo, a agotar nuestros recursos naturales y a contaminar más nuestros desequilibrados sistemas ecológicos. Hemos llegado al punto de convertirnos en los basureros del primer mundo. Nuestra pobreza es tal y nuestros hábitos de consumo están tan distorsionados, que "reciclamos" los desechos del primer mundo: usamos llantas usadas, autos usados, zapatos usados, ropa usada y hasta medicinas y alimentos vencidos.

Pretender ingenuamente que podemos desarroIlarnos siguiendo los patrones capitalistas del desarrollo es una vana ilusión. Si desde el punto de vista histórico ello no fue posible en el pasado, cuando las condiciones internacionales eran menos desfavorables, persistir en los mismo no sólo demuestra ceguera histórica, sino una terquedad ideológica hipócrita e interesada. El que a unos pocos les vaya bien con el sistema, no es garantía de que el sistema pueda asegurarle mejores condiciones de vida a las mayorías populares. Es más, si unos viven

32. El País Digital. 3 de mayo de 2000.

33. Ibid.

34. Fortune, julio, 2000.

35. El País Digital, 11 de abril de 2000. 
muy bien es porque la gran mayoría vive muy mal.

Finalmente, debemos señalar que otro obstáculo al desarrollo de suma importancia se encuentra en el modelo industrial de crecimiento económico, en que se fundamentan la economía capitalista y el deterioro del medio ambiente que genera. De manera que si en los países subdesarrollados se persiste en buscar crecimiento económico siguiendo los patrones de producción y consumo propios de los países industrializados, no sólo no conseguirán desarrollo social para las personas, sino que acabarán más pronto con el precario

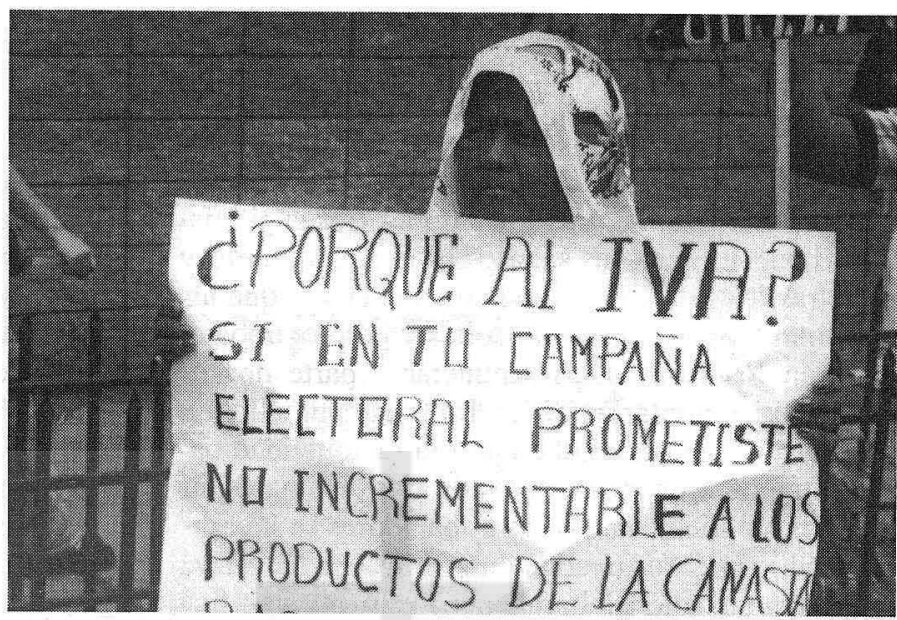
equilibrio ecológico en el cual viven.

\section{Las esperanzas y desesperanzas}

Cuando leemos que uno de los responsables de la profundización de la pobreza, esto es, un alto funcionario del Banco Mundial, reconoce que

Hace más de diez años se tenía la idea de que los expertos teníamos las soluciones; ahora no es así, y esto es importante porque ahora nos damos cuenta que son los pobres mismos quienes deben buscar sus soluciones y que nuestro papel es ayudarles ${ }^{36}$.

Ciertamente, abrigamos alguna esperanza en que se pueda comenzar a enfrentar de manera correcta la problemática de la pobreza, ya que la subjetualidad de los pobres en el proceso para resolver su pobreza es la tesis que hemos venido sosteniendo desde que publicamos nuestro primer trabajo sobre La nueva economía popular, a inicios de la década de los noventa, el cual no partía de especulaciones teóricas, sino de observaciones empíricas; por fortuna para los pobres, la realidad se está imponiendo. Pues, los pobres se han organizado con la finalidad de enfrentar su pobreza mediante la realización de actividades económicas. Ellos son los sujetos de su proyecto, participan activa y solidariamente, y hacen propuestas más que protestas. Los pobres, haciendo más que pidiendo, han sido quienes han logrado encontrar la solución a sus problemas de pobreza. Ellos, los propios pobres y no los teóricos, los técnicos, los

doctos y brillantes genios de la economía, han sido quienes han proporcionado una brillante respuesta práctica al intrincado problema teórico de cómo resolver la problemática de la pobreza.

Ejemplos sobre experiencias exitosas de combate contra la pobreza abundan en nuestro país y en el mundo entero, y lo que las caracteriza a todas, además de lo previamente señalado, es que sus actividades las realizan no siguiendo la lógica capitalista, sino una lógica de sobrevivencia y es cuando los asesores y los técnicos pretenden introducirles, artificiosamente, la lógica empresarial capitalista que sus procesos fracasan.

Si realmente deseamos enfrentar la pobreza en el capitalismo de manera eficaz y eficiente, es preciso respetar la lógica que anima a los pobres en sus actividades económicas, las cuales no se fundamentan en la productividad ni en el consumismo, sino que descansan en lo que Ellacuría denominaba civilización de la pobreza, la cual tiene la ventaja adicional de ser una alternativa de vida conforme con el equilibrio ecológico.

Ciertamente, es un riesgo para el sistema capitalista el que aparezca en sus entrañas otro sistema de organización social, económico y cultural, que demuestre su funcionalidad. Pero resulta más práctico que vivir con el temor a los pobres y con la amenaza siempre presente de que, en determinado momento, pueda ocurrir una explosión social.

36. Así se expresó Ferranti, Vicepresidente para América Latina y el Caribe del Banco Mundial, cuando visitaba nucstro país, en marzo de este año. 
Vivir en residenciales fortificadas, pagando seguridad y con el temor a que algo pudiera ocurrirle a los hijos, es algo que perfectamente podría evitarse al disminuir las diferencias abismales que, en materia de riqueza, actualmente existen. Pero ello no ocurrirá mientras los empresarios capitalistas no paguen mejores salarios a sus trabajadores y mientras los pobres no cuenten con activos que les permitan ganarse la vida con su propio trabajo. ¿Podrán los capitalistas renunciar a parte de sus ganancias voluntariamente? En realidad, ello no parece ser posible atendiendo a la racionalidad capitalista; sin embargo, sí ha sido posible conseguir mejores salarios cuando los trabajadores se han organizado. Desafortunadamente, hoy en día, priva la desorganización laboral, pero esto no significa que siempre tenga que ser así. En cuanto al acceso que deben tener los pobres a los activos, es de suma importancia evitar los errores propios de los gobiernos populistas y encontrar nuevas modalidades que, sin tener que acudir a las nacionalizaciones o confiscaciones, posibiliten a los pobres el acceso a recursos económicos. Aquí es donde se vuelven muy valiosas las experiencias exitosas del combate contra la pobreza.

Sin embargo, también hay desesperanza, porque vivimos en un mundo dominado por las ideas neoliberales. Para mostrar un ejemplo de cómo piensan estas personas, citamos a un economista español, catedrático en Barcelona y Nueva York, y colaborador del Fondo Monetario Internacional, con fama de ultraliberal irreductible, quien sostiene:

hay que saber que los afectados por la pobreza extrema no tienen siquiera la capacidad inteleclual para pensar en estos problemas. Desde ese punto de vista, tenemos que ser nosotros, que hemos tenido el privilegio de estudiar en países ricos, quienes analicemos estos problemas ${ }^{37}$.

Tal ceguera intelectual sólo es comprensible si reconocemos que la ideología es capaz de obnubilar hasta los más brillantes intelectos y que el neoliberalismo, con su endiosamiento del mercado, más que una teoría económica es una ideolo- gía al servicio de los miserables intereses de los propietarios de las grandes empresas transnacionales, quienes siendo tan fuertes como lo son, claman por la libertad comercial a sabiendas de que cuentan con todas las ventajas para salir triunfantes, aun a costa de sus mismos hermanos de clase. La burguesía, sólo cuando se ve amenazada como clase, acude a la solidaridad de clase, pero cuando se trata de las luchas comerciales, animadas por el interés económico, no respeta ni a su misma madre. Negocios son negocios, ésa es su consigna; lo de libertad, igualdad y fraternidad estuvo muy bien mientras había que acabar con la clase feudal y se necesitaba del apoyo de los trabajadores, pero una vez que detentan el poder, devoran literalmente a sus antiguos aliados.

\section{A manera de conclusión}

Si bien el sistema capitalista tiene para rato, su mayor contradicción se encuentra en sus tendencias, las cuales apuntan a la disminución cada vez mayor de los consumidores efectivos, y si no existen consumidores reales, esto es, personas con poder de compra, no pueden existir empresarios capitalistas.

En consecuencia, la mejor manera de prolongarle la vida al sistema es disminuyendo la pobreza y la exclusión social, lo cual demuestra con claridad el interés de los organismos internacionales por enfrentar ambos problemas. No se crea, pues, que el capitalismo se ha humanizado o que los empresarios se han sensibilizado y, con ellos, sus intelectuales orgánicos, o que la solidaridad internacional responde a principios humanistas. Esta situación, sin embargo, no niega que haya personas bien intencionadas, que por razones éticas o cristianas sean solidarias con los pobres.

Bien, para concluir, nada más deseo que a quien lea este artículo le quede el mismo sabor amargo en la boca que yo experimenté cuando lo escribí, a causa de lo mal que anda el mundo debido a la racionalidad capitalista, que anima a nuestra sociedad globalizada. 